\title{
El poder de la estructura: Edificio BBK paraninfo (Bilbao)
}

\author{
The power of the structure: The "BBK" auditorium (Bilbao) \\ R. Losada(*), E. Egia(**), E. Rojí(*), J. Cuadrado(*)
}

\section{RESUMEN}

La última obra terminada por Álvaro Siza en España permite reflexionar sobre el valor del sistema estructural en la composición de formas y huecos, de vuelos y los movimientos de alineación de fachadas; en suma, de la creación del espacio arquitectónico y su envolvente.

El edificio BBK Paraninfo muestra un sistema estructural de láminas-muro, con forjados de estructura mixta (acero-hormigón) y cimentación de losa de hormigón armado sobre pilotes barrenados.

La posición y dimensiones de huecos sin apoyos intermedios y las características de los vuelos dan lugar a una discontinuidad de transmisión de acciones, que requieren fuertes armaduras en secciones limitadas de hormigón, comportando una complejidad de ejecución y actuación frente a retracciones del hormigón.

Arquitectónicamente, la estructura pasa totalmente desapercibida en una arquitectura caracterizada por su simplicidad visual, complejidad espacial y constructiva, donde la luz natural y sus matices juegan un papel básico para la definición de espacios.

405-7

Palabras clave: Siza; láminas-muro; estructura portante; pilotes barrenados.

\section{SUMMARY}

The most recently work of Álvaro Siza, in Spain, shows us the merits of the structural system in the composition of its forms and spaces, its overhangs and the movement in the alignment of its façades; the creation of the architectonic space and its envelope.

The BBK Auditorium consists of a structural system of layered walls, mixed structural slabs (steel, concrete) and reinforced concrete slab foundations on augercast piles.

The position and dimensions of the spaces with no intermediary supports and the characteristics of the overhangs create discontinuous transmission of actions, which require strong reinforcements in limited sections of the concrete, implying complexity in its execution and performance in response to concrete shrinkage.

Architecturally, the structure goes entirely unnoticed in the architecture, characterized by its visual simplicity within an intricate spatial and constructive arrangement, where the nuances of natural light play a basic role in the definition of the spaces.

Keywords: Siza; layered walls; load-bearing structure; augercast piles.

$\left(^{*}\right)$ Universidad del País Vasco/Euskal Herriko Unibertsitatea, Bilbao (España) 
1. Plano de localización del edificio.

2. Estética del edificio acabado.

\section{INTRODUCCIÓN}

En el proceso de terciarización de la otrora industrial Villa de Bilbao, la zona de Abandoibarra ha jugado un papel trascendental. La ría navegable, sus muelles, unido a los almacenes y tinglados, han dado paso a la reordenación y urbanización de los espacios liberados en el tiempo, posibilitando que el Ensanche bilbaíno se acerque a la ría de Nervión (1). La participación de profesionales de reconocida valía en el proceso ha supuesto un catálogo de "arquitecturas" varias, que se iniciaron con el Museo Guggenheim de Frank Gehry (Figura 1).

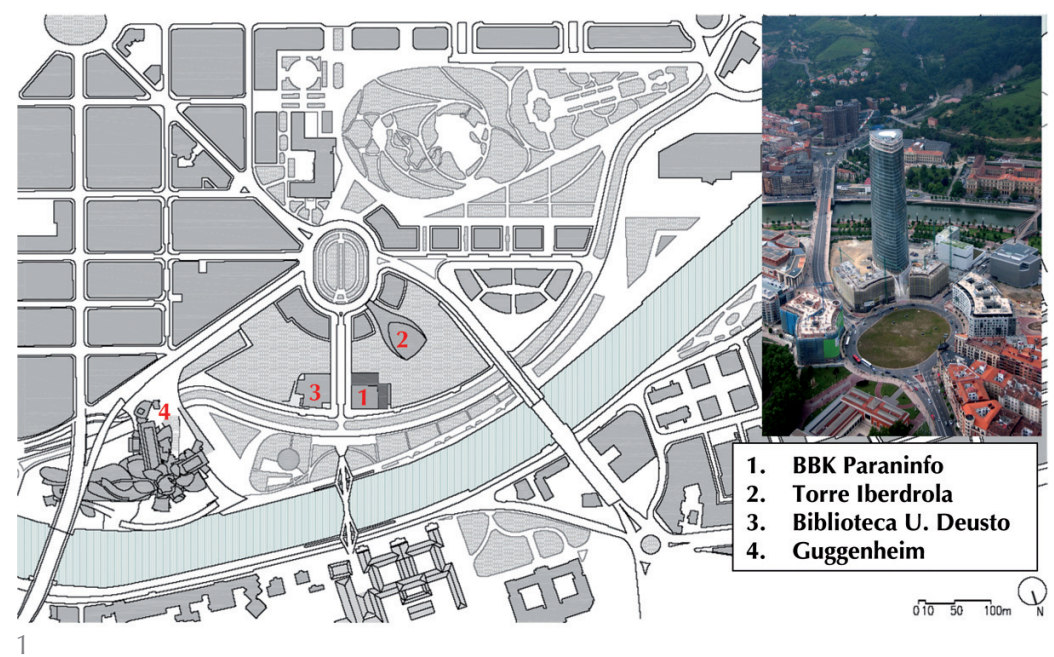

Álvaro Siza, arquitecto portugués reconocido entre otros méritos, por contar con el Premio Pritzker, también ha sido Ilamado a participar en este nuevo modelo de ciudad. Invitado por la Bilbao Bizkaia Kutxa (BBK) se ha hecho cargo de una de las últimas piezas del puzzle; precisamente, la colindante a la Torre Iberdrola de Cesar Pelli y a la Biblioteca de la Universidad de Deusto, obra de Rafael Moneo, configurando el eje viario que conduce hacia el Ensanche.

El planeamiento urbanístico había concebido en ese punto dos edificios simétricos, en cada alineación de la calle, con cornisas enrasadas a modo de puerta urbana. Moneo ha concebido un edificio, compacto en su volumen edificatorio, con una envolvente de vidrio (pavés). Siza, sin embargo, de alguna manera ha jugado con el negativo del volumen. Ha concebido un edificio que si bien en planta baja reproduce la ocupación máxima urbanística, en las plantas altas se transforma en un edificio en forma de " $\mathrm{L}$ " mostrando su concavidad hacia el Museo Guggenheim, en un permanente diálogo arquitectónico entre ambas obras. Una forma en "L" cuya piel en la fachada cóncava está tratada como envolvente pegada (sistema de aislamiento de fachada por el exterior), con recubrimiento de azulejo gris, que según la incidencia de la luz, muestra irisaciones y diferentes tonalidades. Sin embargo, la fachada convexa tiene un tratamiento de envolvente ventilada, con un majestuoso despiece de aplacado de mármol Macael (Figura 2).

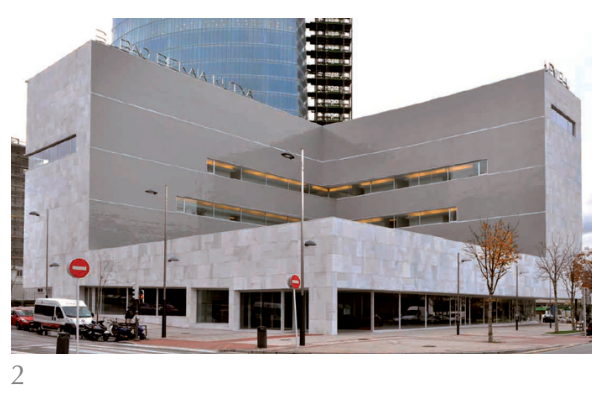

\section{DESCRIPCIÓN DEL EDIFICIO}

El edificio se concibe para albergar lo que será el Paraninfo de la Universidad del País Vasco (UPV/EHU), junto con una serie de salas a ocupar por actividades museísticas, los despachos del Rector y Vicerrector y otras dependencias auxiliares destinadas a tienda y a la sede del Instituto de Euskera.

Siza ha desarrollado el Programa en una construcción con planta sótano, donde se albergan instalaciones y un aparcamiento de vehículos para 31 plazas. Ocupa prácticamente la totalidad de la parcela, accediéndose al sótano a través de una rampa rectilínea adosada al edificio, pero integrada a éste mediante una potente marquesina.

La planta baja recoge en su parte central el local destinado a paraninfo, disponiendo en su frente, totalmente acristalado, un amplio vestíbulo. Lateralmente se localiza la tienda, mientras que en la orientación Oeste, se ubican los aseos, accesos a las plantas altas y dependencias de apoyo al Paraninfo. El frente Sur, prácticamente ciego, es ocupado por el escenario del paraninfo, así como por salas de instalaciones.

Las plantas altas dispuestas en forma de "L", cuyos brazos se orientan al Sur (paralelo a la ría) y al Oeste (perpendicular a la ría) están constituidos por sendos paralelepípedos con anchura entre los 12 y $15 \mathrm{~m}$. respectivamente, a los que se accede por dos núcleos de escaleras y ascensores. Existe un tercer núcleo de accesos verticales localizados junto al vestíbulo principal, pero que sólo comunica la planta sótano y la planta baja.

Las plantas altas están enrasadas en su cornisa, pero el cuerpo Sur presenta 3 plantas altas y el Oeste 4 plantas. Esto hace, que las últimas plantas dedicadas a instalaciones, en el brazo paralelo a la ría (Sur), cuente doble altura. 


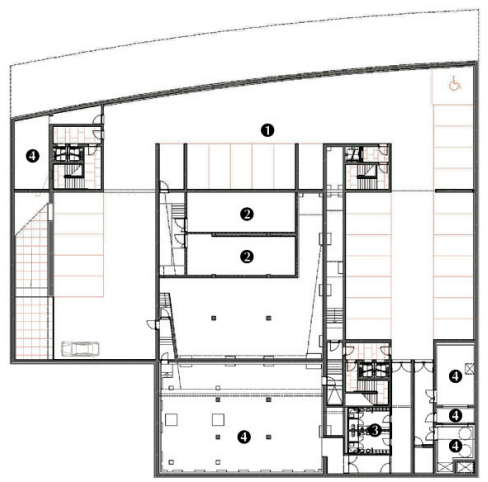

Planta sótano
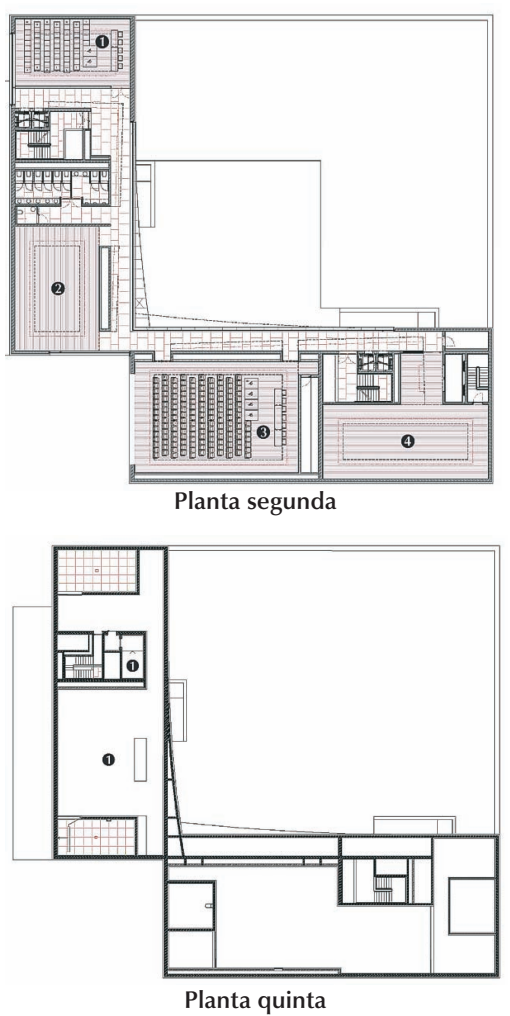

Los extremos de los brazos de la " $\mathrm{L}$ ", en la cubierta, que es plana y ajardinada, están dotados de patios o troneras de una sola planta, lo que permite la toma de aire de las instalaciones de la última planta, que quedan ocultas de vistas desde los edificios circundantes, no apreciándose ningún elemento o máquina sobre el nivel de cubierta.

La disposición interior de las plantas altas, desde los accesos verticales ubicados en los extremos, se configura con espacios en colindancia, repartidos a lo largo de un pasillo que recorre el perímetro exterior de la "L" en la envolvente cóncava.

Esos pasillos se caracterizan en su cerramiento exterior por estar rasgados longitudinalmente de forma continua, posibilitando la iluminación natural hacia el interior. Las dependencias dedicadas a usos museísticos son ciegas y el resto de locales dispone de las imprescindibles aberturas
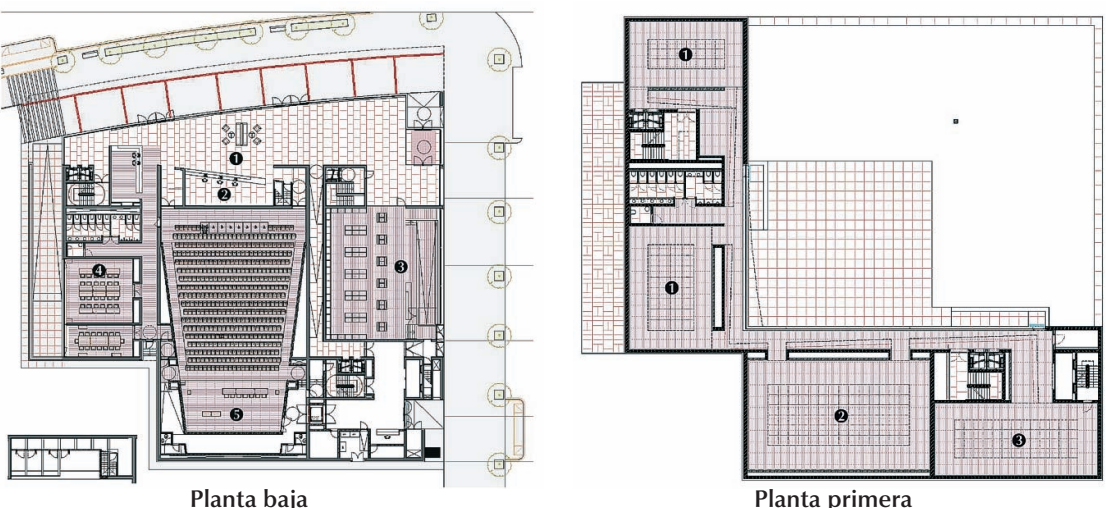

Planta baja
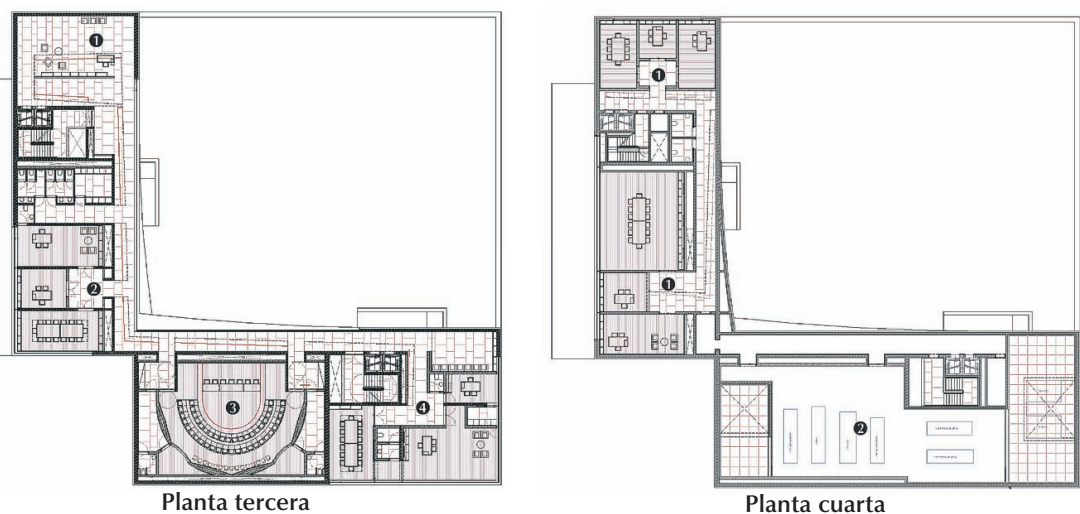

Planta cuarta

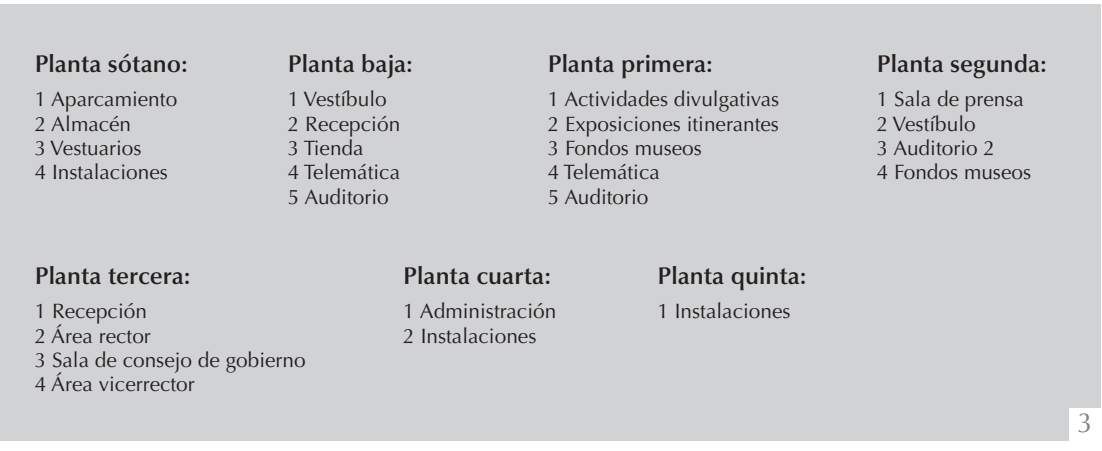

hacia el exterior. El resultado es un edifi-

3. Plantas del edificio. cio con sensación de compacto, incluso se podría pensar que casi ciego y, con ello, a falta de iluminación natural interior. Sin embargo, Siza, auténtico mago en el manejo de la luz, con los pasillos rasgados y las imprescindibles aberturas al exterior, en el resto del edificio, consigue crear un ambiente interior cargado de contrastes de claridad y penumbra, de simplicidad desde la complejidad, mostrando esa sensación y espacios característicos de la arquitectura del portugués.

Desde el punto de vista formal-constructivo debe destacarse el techo de la planta baja, en la fachada a la Avda. Abandoibarra, que conforma un vuelo creciente en un vano de prácticamente $38 \mathrm{~m}$. de longitud, que se remata con el vuelo del brazo Oeste de la "L", generando así una ampliación del espacio público a nivel de calle (Figura 3). 

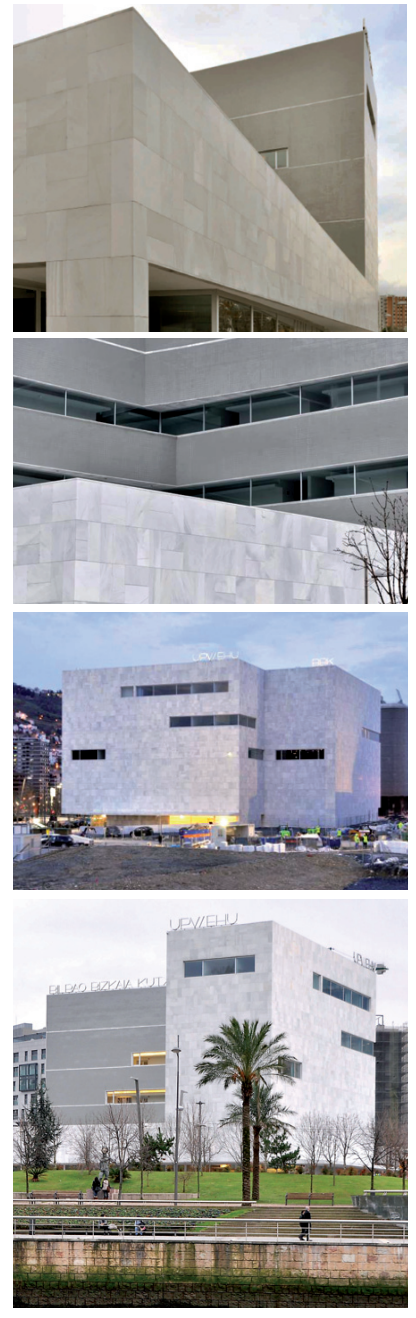

4

4. Detalles de la arquitectura exterior.

5. Detalles de la cimentación.

\subsection{El concepto del edificio y la forma}

La contemplación de las plantas del edificio de Siza nos muestra una funcionalidad dinámica, en tanto que la utilización del edificio parece requerir un continuo deambular a través de sugerentes pasillos alrededor del paraninfo o a lo largo del contorno exterior cóncavo. Esta sensación se remarca con la masividad del edificio, que se refleja en planta con las láminas-muro de hormigón armado, que van configurando los diferentes espacios interiores.

Las técnicas constructivas modernas (a partir del siglo XIX) nos conducen a una progresiva especialización del material en elementos y sistemas dotados de misiones específicas. Con el paso del tiempo la función soporte del edificio y la del cerramiento, progresivamente, se han convertido en sistemas del edificio claramente diferenciados (2). La estructura, como lo hiciera la construcción compacta, deja de simultáneamente vertebrar y resistir acciones, con el mantenimiento o reflejo de la forma y de la envolvente.

Siza, en este edificio, se rebela contra esa actitud proyectual "moderna", basada en el sistema estructural aporticado, proponiendo, una vez más (tal y como ha hecho en la Facultad de Ciencias de la Información en Santiago de Compostela, en el edificio de nuevo auditorio para Ceuta o en el museo para la Fundación Ibêre-Camargo en Porto Alegre), que la forma adquiera entidad arquitectónica, sin obviar sus aspectos constructivos y estructurales (3). Mantiene su principio de composición y diseño en todos los espacios interiores y en el conjunto, quedando reflejado en el más mínimo detalle constructivo del edificio, en un proceso en que la estructura le acompaña fielmente y ajustada a la forma, como si se tratara de un guante respecto a la mano.
La asunción de la estructura a base de elementos laminares portantes y cumplimentando, también, la función de cerramiento, permite configurar los huecos de fachada que parecen, más que cumplimentar la necesidad de iluminar el espacio interior, unos vacíos arrancados o esculpidos en el conjunto tectónico masivo del edificio (4) (Figura 4).

\section{PlANTEAMIENTOS ESTRUCTURALES}

\subsection{Cimentación}

Del estudio geológico-geotécnico se dedujo la existencia de unos rellenos heterogéneos apareciendo, en profundidades entre $16,90 \mathrm{~m}$. y $18,60 \mathrm{~m}$., un sustrato rocoso de margocalizas grises con resistencia a compresión simple $\sigma_{\mathrm{u}}=2,5 \mathrm{kN} / \mathrm{cm}^{2}$. Sobre dicho sustrato se encontraron fangos arenosos, limos y arcillas, así como arenas.

La profundidad del nivel freático variaba entre 2,5 m. y 7,5 m., lo que en el caso más favorable venía a coincidir en unos $60 \mathrm{~cm}$. u $80 \mathrm{~cm}$. por encima del nivel de excavación, aconsejando el disponer de material drenante bajo la losa de sótano.

La cimentación del edificio es del tipo profundo mediante pilotes in situ, empotrados en roca (16 m. bajo el nivel del suelo del sótano).

Se han ejecutado pilotes barrenados in situ, sin camisa y con barrena continua, con control de calidad en tiempo real de la ejecución, tanto en la fase de la perforación como en la de hormigonado, consiguiendo así un tope estructural del pilote a cargas verticales de $60 \mathrm{~kg} / \mathrm{cm}^{2}$, permitiendo aprovechar mejor las características resistentes del hormigón utilizado (Figura 5).

Las características principales de los elementos pilotados han sido las siguientes:
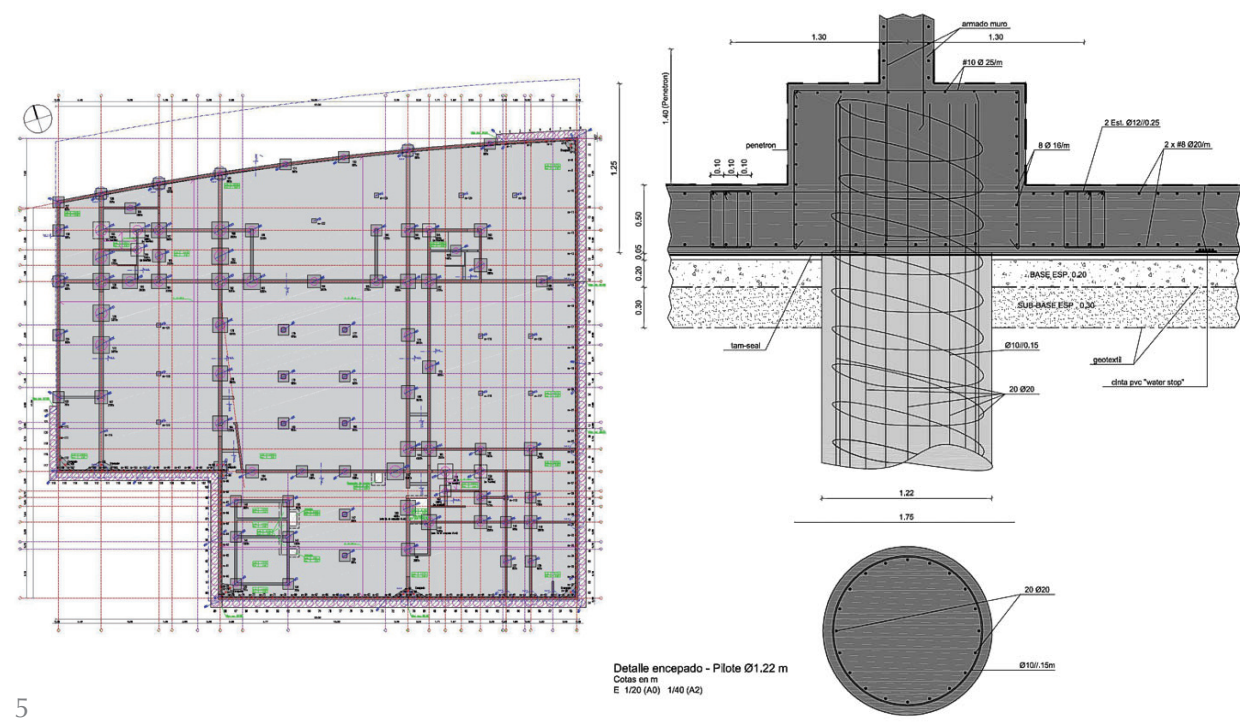

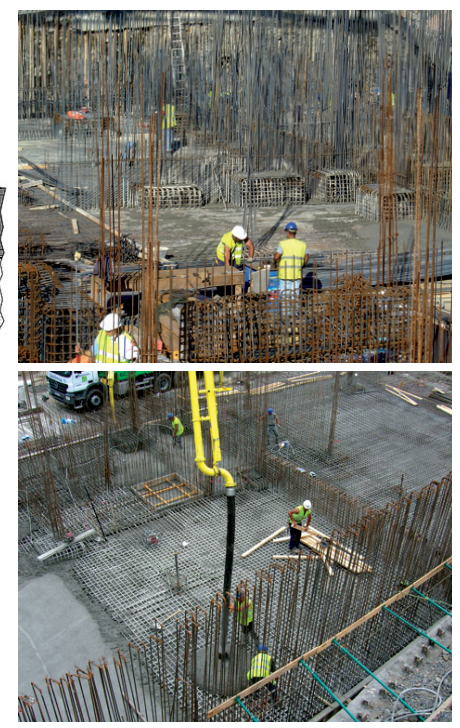


- Todos los elementos estructurales laminares están cimentados sobre pilotes aislados de diámetro variable de 620, 820, 1.020 y $1.220 \mathrm{~mm}$., en función de las diferentes solicitaciones, trabajando a compresión con cuantías del 0,4\%.

- Los pilotes están enlazados con una losa de hormigón armado a nivel del suelo de sótano de $50 \mathrm{~cm}$. de espesor, que tiene funciones de arriostramiento para corregir excentricidades o pequeños desplazamientos de la posición teórica del pilote y capacidad para soportar posibles sub-presiones, originadas por un nivel freático de 1,2 m. por encima de la cota superior de la misma.

\subsection{Muros de sótano}

Las condiciones del entorno determinaron las diferentes soluciones que se dieron a los muros de sótano.

En dos de los frentes fue posible realizar una excavación con cierto talud, sujetando de forma provisional el terreno mediante la hinca de raíles. Esto permitió que se ejecutaran pilotes cada $5 \mathrm{~m}$., empotrados en roca, descabezándose a nivel del suelo del sótano. Realizados los encepados invertidos se enlazaron con la losa y, sobre ellos, se apoyaron los muros del sótano, que se ejecutaron encofrando a dos caras.

En la alineación Este, para poder realizar la excavación sin que se movieran la calle, ni los rellenos sobre columnas de grava que existen, se ejecutaron pantallas de pilotes in situ de $82 \mathrm{~cm}$. de diámetro separados a ejes $110 \mathrm{~cm}$. Se desechó la ejecución de una pantalla continua, pues se temía que los lodos tixotrópicos no pudieran sujetar la excavación, debido a las características del terreno: rellenos muy permeables.

La altura máxima de la excavación y, por tanto, de la contención fue de $7 \mathrm{~m}$., incluyendo el espesor de la losa de cimentación $(50 \mathrm{~cm}$.) y el espesor de terreno mejorado $(50 \mathrm{~cm}$.).

Los pilotes de la pantalla se hicieron trabajar en ménsula (flexión), empotrados en el terreno una vez realizada la excavación, estando fuertemente armados (cuantía próxima al $2 \%$ ). En la esquina Sureste, con mayor altura de excavación, se apuntaló la pantalla a la altura de la viga de coronación; una pantalla contra la otra mediante perfiles arriostrados de acero.

Lo habitual es que el sistema de contención utilizado para realizar la excavación de un sótano sirva de cimentación de los pilares perimetrales de un edificio. En este caso, las láminas-muro resistentes debieron apo- yarse en una pantalla de pilotes (diámetro $82 \mathrm{~cm}$.) totalmente excéntrica, al no poder ocupar ésta más espacio que el proyectado en el sótano por necesidades funcionales del aparcamiento.

Consecuentemente, la carga de las láminasmuro de este perímetro se transmite a la pantalla de pilotes con grandes excentricidades (50 y $55 \mathrm{~cm}$.), que provocan una flexión que no se podía resistir con los espesores de 30 y $40 \mathrm{~cm}$. dispuestos desde la arquitectura. Estas circunstancias Ilevaron a que las láminas-muro resistentes, en estos frentes donde se han ejecutado pantallas de pilotes, se soporten directamente sobre micropilotes (diámetro $210 \mathrm{~mm}$.) empotrados en roca con la cabeza al nivel del suelo del sótano, Colocados en los límites de la losa (Figura 6).
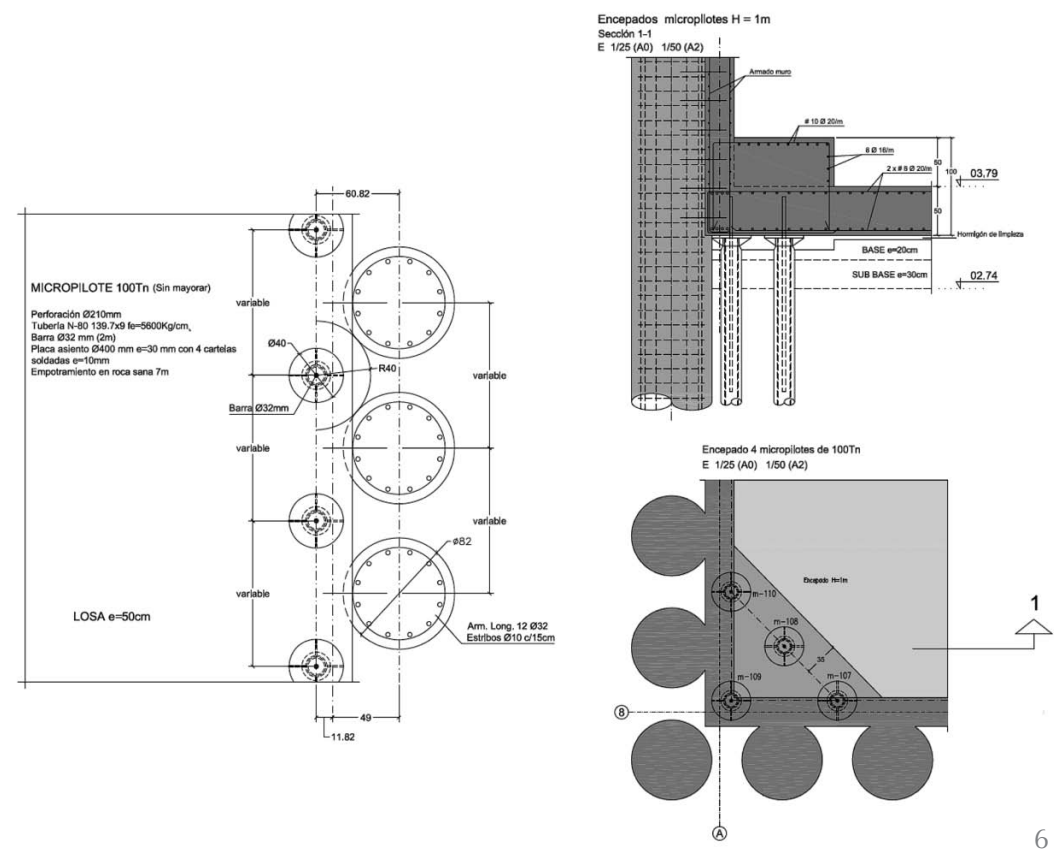

Las láminas se conectan en el sótano con los pilotes de la pantalla y se hormigonan con parte de la viga de coronación de la pantalla de pilotes.

\subsection{Estructura}

La estructura se ha ejecutado con elementos verticales laminares de hormigón armado (muros) en dos direcciones ortogonales y forjados de losas macizas con estructura mixta, que aseguran la estabilidad y rigidez del edificio. Salvo los pilares que soportan la losa del suelo del paraninfo (escenario y zona de butacas), en planta sótano, el sistema estructural carece de pilares.

La distribución de muros resistentes en ambas direcciones, acomodada a los espacios arquitectónicos, arriostrados con los forjados, consigue que cada muro trabaje principalmente con esfuerzos en su plano, obteniéndose así espesores de 20,30, y $40 \mathrm{~cm}$.
6. Micropilotes de apoyo de muros de sótano.

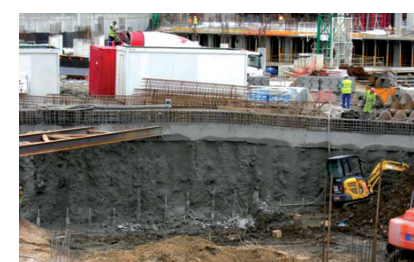



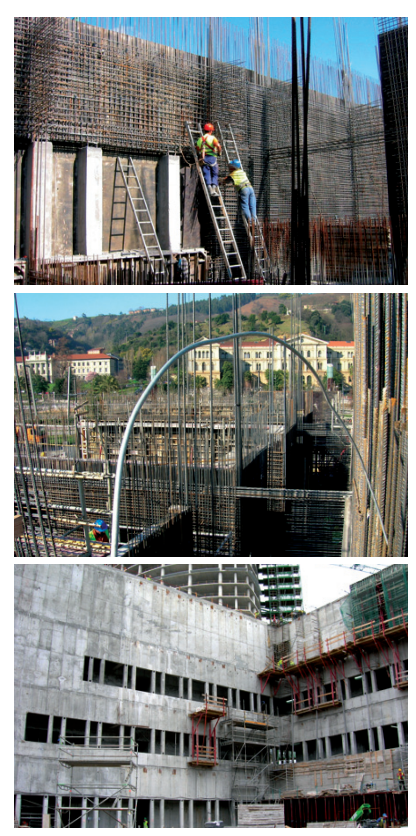

Ejecución de la estructura de hormigón.

8. Montaje y detalles de los forjados mixtos
En el esquema estructural unos muros son las líneas de carga principales del edificio (con espesores de 30 y $40 \mathrm{~cm}$.) y, otros, los que conformando las distribuciones interiores a modo de particiones de hormigón armado (con espesores de 15 y $20 \mathrm{~cm}$.) son necesarios para conseguir la rigidez del conjunto.

La discontinuidad superficial de algunas de las láminas - muro como consecuencia de los huecos rasgados y su trabajo en vuelo, motivó la necesidad de disponer durante la obra, unos apuntalamientos metálicos hasta la losa de cimentación y encepados de pilotes, que mantuvieran la estabilidad del plano, en tanto se completaba el hormigonado y fraguado del hormigón del muro, así como la capacidad resistente del elemento.

Igualmente, el rasgado de los huecos, muy especialmente a lo largo de los pasillos hacia las fachadas cóncavas de la "L", obligaron a disponer modularmente unos pilares de hormigón armado provisionales, que sirvieron de apoyo a los encofrados trepantes utilizados en la ejecución de las láminas-muro (Figura 7).

\subsubsection{Forjados mixtos}

La mayor parte de los forjados del edificio tienen grandes luces, 12 y $13 \mathrm{~m}$ en las plantas altas correspondientes al cuerpo constructivo en forma de "L" y hasta $20 \mathrm{~m}$. en el techo de la planta baja, que da lugar a la terraza ajardinada, por lo que se recurre a una solución de estructura mixta; es decir, vigas metálicas con perfiles desde HEB 320 a HEB 700 (acero de calidad JO producido en Luxemburgo), separadas 1,50 m aproximadamente y conectadas con pernos de rasante a losas de hormigón armado de $15 \mathrm{~cm}$. de espesor.
La losa de los forjados mixtos se armaba fuertemente sobre las alas de las vigas metálicas (8Ø25 ó 8Ø20). De esta manera, se conseguía una mayor capacidad de los forjados mixtos en el centro del vano, que se plantearon contraflechados, trabajando esta armadura a compresión junto a la losa.

El enlace de los forjados mixtos en los muros consistió en anclar la armadura de la losa y sus refuerzos (8Ø25 ó 8Ø20), y soldar las vigas metálicas en todo el perímetro a las placas de anclaje, previamente embebidas en el muro.

La placa a la que se suelda la viga metálica es la placa interior de un "bocadillo" de chapas colocadas a ambas caras del muro. Estas placas están unidas entre sí con barras soldadas en agujeros avellanados a la altura de las alas de las vigas metálicas. De esta forma, el anclaje de la placa interior se consigue a través del tope, que hace la placa exterior contra el hormigón.

Con estos detalles constructivos el forjado tiene capacidad para soportar cierto momento de empotramiento en su enlace con los muros. El conjunto formado por el forjado mixto y los muros de apoyo tiene igualmente cierta capacidad para soportar esfuerzos horizontales perpendiculares a los muros (Figura 8).

\subsection{Consideraciones constructivas sobre los cálculos estructurales}

El edificio se ha considerado sometido a las acciones indicadas en el Código Técnico de la Edificación (5). Dado el carácter masivo de la estructura de hormigón armado, las
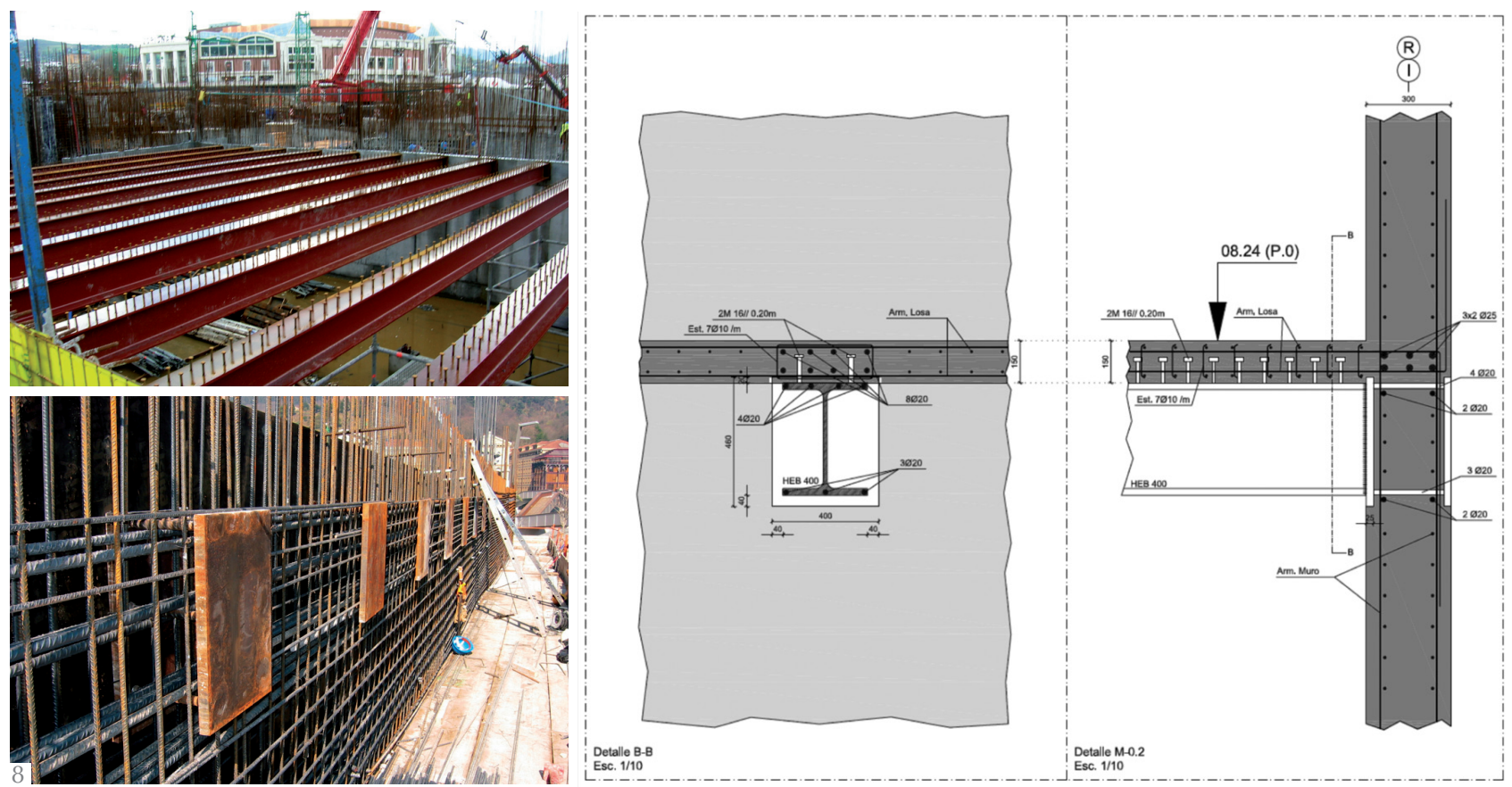
acciones reológicas han requerido la adopción de medidas constructivas específicas.

Se trata de un edificio muy rígido, sin juntas por razones arquitectónicas, con una dimensión máxima en planta de 55 m.

Se ha considerado un valor de retracción a plazo infinito de $0,25 \mathrm{~mm} . / \mathrm{m}$. $(0,25 \mathrm{~mm} . / \mathrm{m}$. x $55 \mathrm{~m} .=14 \mathrm{~mm}$.).

La mayor parte de la retracción se produce en edades tempranas de hormigón; por ello, a estos efectos los primeros $50 \mathrm{~cm}$. de los muros en cada planta llevan una armadura horizontal adicional.

También se han estudiado con especial atención las acciones debidas al viento y aquellas otras internas que originan los cambios de temperatura. A este último respecto se han considerado cambios de temperatura de $15^{\circ}$.

Con carácter general se han utilizado los métodos de cálculo de Estados Límite últimos: de equilibrio, agotamiento de la sección, inestabilidad, anclaje y adherencia, así como de Estados límite de servicio: fisuración, deformación y vibraciones.

Se ha previsto la aparición de fisuras en el hormigón, lo que no supone ningún inconveniente para su normal utilización, siempre que quede limitada la abertura máxima de fisura a valores compatibles con las exigencias de durabilidad, funcionalidad, estanqueidad y apariencia.

El ambiente previsto para la estructura es IIla (exposición en ambiente marino aéreo) (6). Se han definido, además, los recubrimientos de la armadura y las características mínimas del hormigón, con un límite de abertura de fisura, Wmáx $=0,2 \mathrm{~mm}$., para proteger la armadura de la corrosión por cloruros.

Sin tener en cuenta ningún esfuerzo más, como pueden ser la tracción en tirantes o la flexión, la consideración solamente de la retracción, el descenso de temperatura y el control de la fisuración hace que cualquier sección deba estar fuertemente armada.

\subsubsection{Elementos singulares de la estructura}

Para facilitar la identificación de la estructura se ha procedido a denominar los elementos que la componen en función del eje en que se configuran. Los ejes perpendiculares a la Ría (orientación Norte-Sur) se han designado por letras mayúsculas a partir de la orientación Oeste; los transversales (orientación Este-Oeste) con números, iniciándose en la fachada Sur.

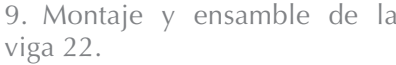

La terraza de la planta primera está volada respecto al cierre de planta baja para lo cual se ha dispuesto de una viga de aproximadamente $38 \mathrm{~m}$. de luz, que recoge el forjado de estructura mixta y que se apoya en un extremo en un muro-pilar (alineación R) $y$, en el otro, en la parte inferior de una gran ménsula que forma parte de una de las láminas verticales resistentes (alineación E).

Esta viga especial está resuelta también con estructura mixta. Se ha configurado como una viga Vierendeel de perfiles metálicos rigidizada con un alma de hormigón armado de $15 \mathrm{~cm}$. de espesor. Se ha construido en taller y ha sido transportada en dos tramos que se han soldado en obra, montándose con la ayuda de dos grúas sobre una cimbra que posibilitaba los $12 \mathrm{~cm}$. de contraflecha que debía disponer. Todas las soldaduras se controlaron mediante radiografías y además se reforzaron las alas de los cordones HEM 550 soldando cubrejuntas por las caras interiores, por haber tenido que realizar la unión en las secciones más comprometidas.

Tiene $4 \mathrm{~m}$ de canto; el cordón superior y el inferior son perfiles HEM 550 tumbados o colocados en horizontal; los montantes HEB 300, separados en general 2,18 m. reduciéndose a 1,43 $\mathrm{m}$. en el apoyo $(\mathrm{R})$ y a $1,13 \mathrm{~m}$. en el apoyo (E).

Para conectar la viga metálica con el alma de hormigón, en todo el contorno, limitado por los cordones superior e inferior y los montantes, lleva conectores M16 cada $10 \mathrm{~cm}$ en los perfiles metálicos, y además toda la armadura del alma, dos mallas \# 10 $\varnothing 10 /$ m., está soldada a dichos perfiles. Así, el alma de hormigón armado forma un diafragma que rigidiza la viga como las diagonales cruzadas de una celosía formando bielas (compresión) y tirantes (tracción).

El forjado mixto de la terraza de planta $1^{\mathrm{a}}$ entrega las vigas metálicas (HEB 700) en el punto medio de los montantes (HEB 300) y, de este modo, el alma de hormigón armado de la viga se enlaza a media altura con la losa del forjado mixto formando paños de $2 \times 2 \mathrm{~m}^{2}$ aproximadamente, de modo que la propia viga hace de antepecho de la terraza.

Las vigas metálicas HEB 700 de 9,5 m de luz se apoyan en los montantes HEB 300 Para ello, se ha soldado solamente el alma, con el fin de evitar un empotramiento que torsionaría la viga. La losa de $15 \mathrm{~cm}$. de espesor del forjado mixto de planta $1^{\mathrm{a}}$ rigidiza el alma de la viga, reduciendo el tamaño de los paños (Figura 9).

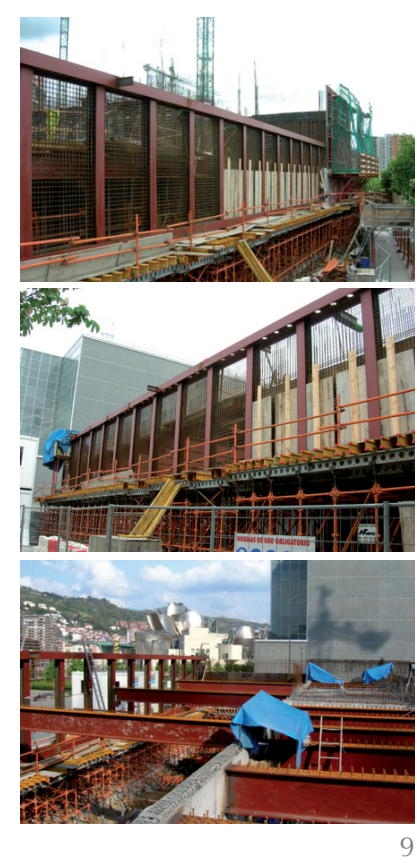


10. Secciones estructurales E y R.
Para retirar la cimbra de la viga, se cuidó la puesta en carga, descimbrando gradualmente y en fases sucesivas. En cada fase se indicó que se descendiese $5 \mathrm{~mm}$. el puntal del centro de la viga $y$, de forma simétrica respecto al centro, se fueran dando descensos variables y proporcionales a $5 \mathrm{~mm}$. en el centro y $0 \mathrm{~mm}$. en los apoyos.

En el momento de quitar la cimbra, la carga que soportaba la viga era inferior a las $10,9 \mathrm{~T} / \mathrm{m}$, puesto que no tenía aún la carga del pavimento de la terraza, ni la sobrecarga, ni el revestimiento de la viga. Se esperaba un descenso de 1,5 cm., con una estimación de la carga existente de un $45 \%$ de la total.

Se midieron los descensos en distintos puntos de la viga en los días siguientes y los desplazamientos fueron muy pequeños, hasta estabilizarse el descenso máximo en $1 \mathrm{~cm}$.

\section{LÁMINAS}

Dadas la posición y características de los huecos dispuestos en las láminas-muro, las secciones más importantes estructuralmente serían: 1, 8, 9, B, E y R (Figura 10).

Para el cálculo de las láminas se ha utilizado el programa ROBOT Structural Analysis. EI armado adoptado por el programa es el mínimo (suma mínima del área $A x+A y)$.

Para calcular las armaduras, el programa permite limitar el conjunto de esfuerzos internos considerados. Los cálculos de armados pueden ser efectuados para el conjunto completo de esfuerzos de lámina (flexión y esfuerzos de membrana), sólo para momentos flectores (flexión simple) o sólo para esfuerzos de membrana.
El armado teórico de las láminas se ha realizado con todos los esfuerzos internos de lámina (de flexión y membrana), siguiendo el método analítico. Estas armaduras teóricas no incluyen las armaduras necesarias de retracción, fisuración y mínimas que garanticen una rotura dúctil.

La comprobación final de la armadura de las láminas verticales se ha realizado con modelos parciales de bielas y tirantes orientados según las direcciones de tensiones principales obtenidas por el programa, así como elementos tipo viga claramente definidos en las láminas. En esta comprobación se simplifica el esquema estructural, del lado de la seguridad, despreciando la colaboración de los elementos estructurales secundarios.

El programa realiza todas las combinaciones necesarias según el CTE para estados límites últimos y para estados límite de servicio (Figura 11).

\section{A MODO DE CONCLUSION: LA IDEA DEL EDIFICIO Y LA ESTRUCTURA}

Siza mantiene en sus proyectos una relación directa con sus colaboradores de las distintas ramas de la ingeniería. Los espacios y formas que diseña deben tener la estabilidad y la seguridad que brinda el sistema estructural, pero éste no debe evidenciarse por sí, sino mostrarse por sus bondades (prestaciones).

De este modo, el sistema estructural, basado en láminas-muros estructurales Ilevados a unos límites tensionales insospechados y a una complejidad de ejecución enorme, dan rotundidad a una obra masiva, que adquiere su condición de objeto de contemplación con la posición y dimensiones de los huecos y vuelos de sus fachadas, obligán-

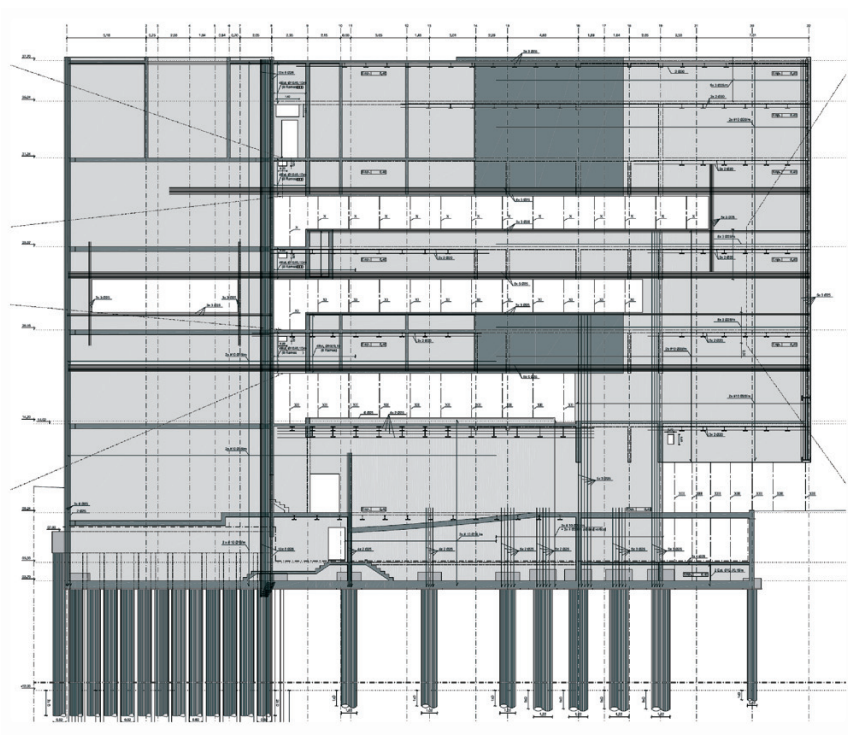

10

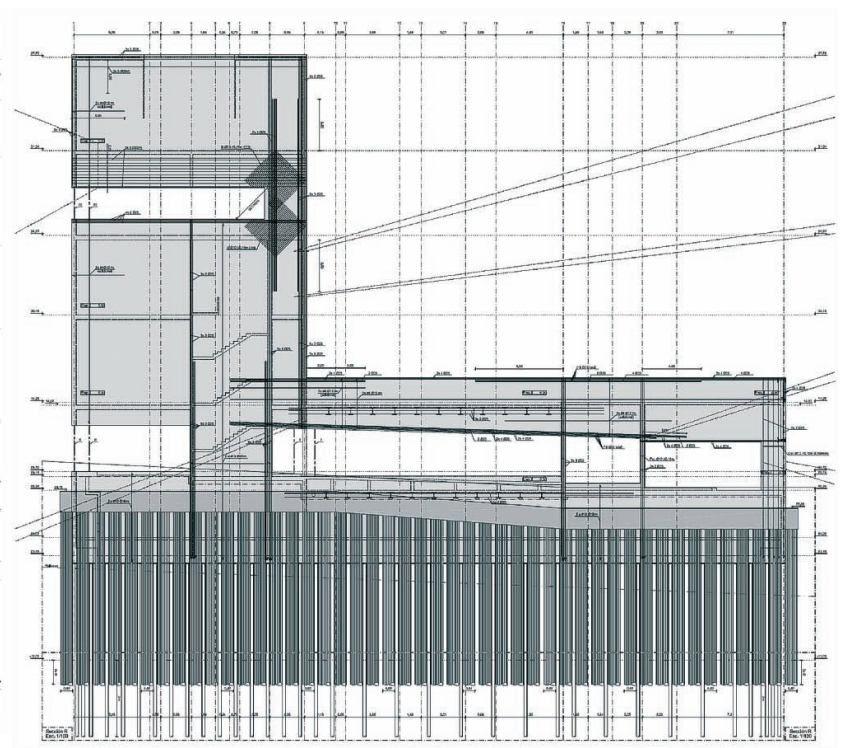

Sección R 
Alineación $E$ :

Armadura horizontal cara exterior:

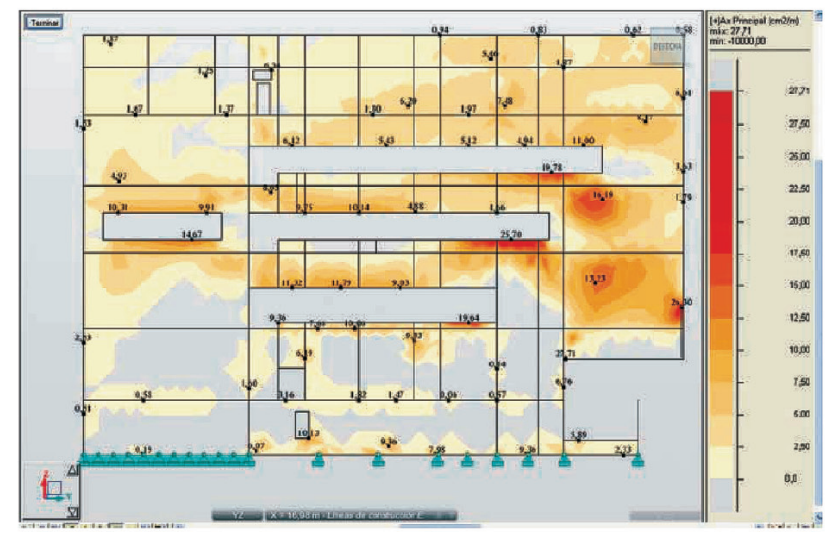

Alineación $E$ :

Armadura horizontal cara interior:

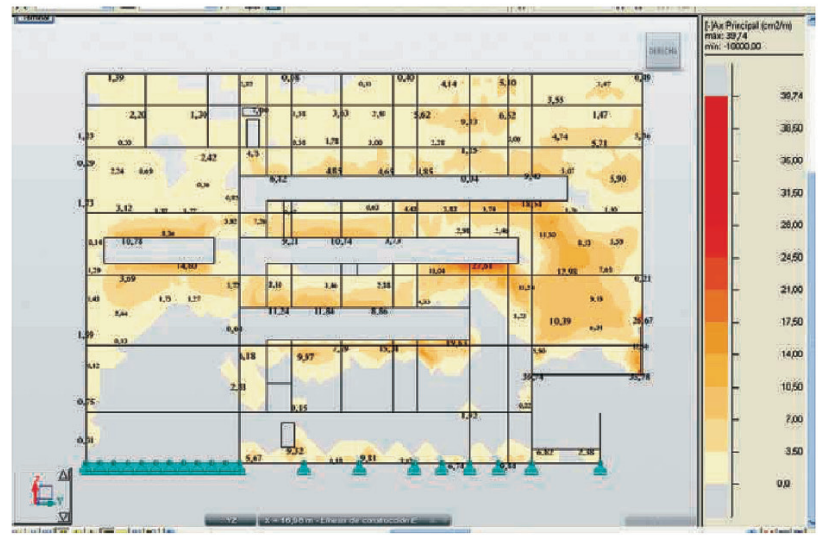

Alineación $R$ :

Armadura vertical cara exterior:

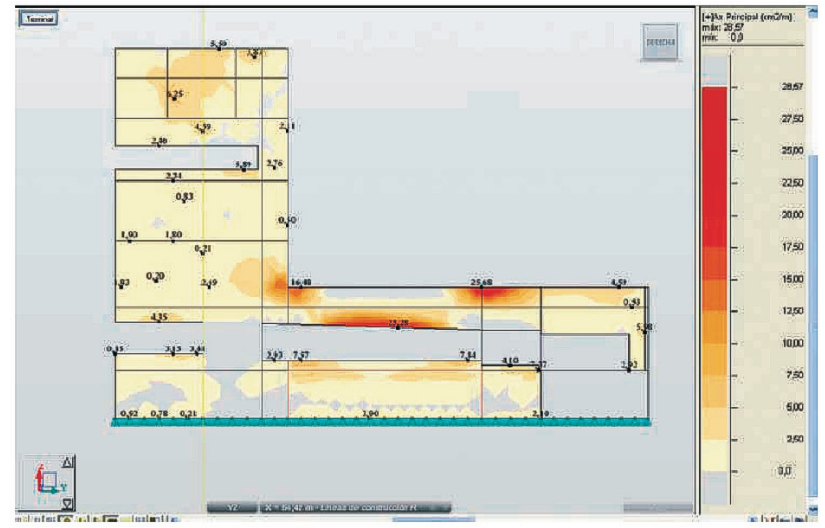

Alineación $R$.

Armadura vertical cara interior:

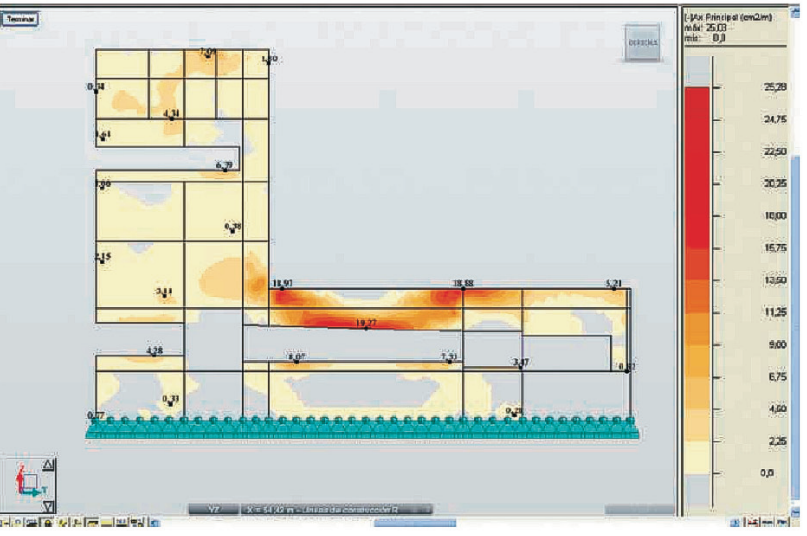

11 donos a mirar ese lugar concreto, por ejemplo, accesos al edificio, vuelo sobre la planta baja, esquinas rasgadas con continuidad sin apoyos intermedios, etc., acentuando nuestra percepción del edificio.
En suma, la estructura cobra su importancia por sus prestaciones de resistencia y estabilidad, así como por remarcar el carácter espacial y masivo de la arquitectura del edificio.
11. Resultados de análisis con ROBOT alineaciones E y R

\section{BIBLIOGRAFÍA}

(1) Ayto. de Bilbao: Plan Especial de Reforma Interior de Abandoibarra. Area de Urbanismo y Medio Ambiente, 1995.

(2) Losada, R. et al.: La envolvente de los edificios: Tipologías y soluciones constructivas p. 2, Publicaciones de la ETSI Bilbao, 2010.

(3) Losada, R.: Escuchando a Alvaro Siza, p. 21, ed. El Autor, 2011.

(4) Siza, A.: The function of beauty. Ed. Phaidon, 2009.

(5) Ministerio de Fomento: CTE DB SE-AE Seguridad Estructural Acciones en la Edificación, 2009

(6) Ministerio de Fomento: EHE-08 Instrucción de hormigón estructural, cap. 2: criterios de seguridad y bases de cálculo, 2008. 